\title{
A HARDWARE PROTOTYPE FOR INTEGRATION, TEST AND VALIDATION OF AVIONIC NETWORKS
}

\author{
José-Philippe Tremblay ${ }^{1}$, \\ Yvon Savaria ${ }^{1}$, Claude Thibeault ${ }^{2}$, \\ Safwen Bouanen ${ }^{2}$ \& Guchuan Zhu ${ }^{1}$
}

1. École Polytechnique de Montréal

2. École de Technologie Supérieure 


\section{Outline}

1. Introduction

2. Network architecture

3. Global approach

4. Implementation details

5. Prototype validation

6. Conclusion 


\section{Introduction}

- Current trends in the avionics domain

-Ever increasing number of functions

-Information flow increase

-Stringent reliability requirements

-Diversity in the transducers market

-Migration to IMA architecture 


\subsection{Introduction}

- Main issues in transducer's integration

-Different types of transducers

-Different communication protocols

-Significant design effort

-Very costly and time consuming

> Solution

-Systematic design approach

-Normalized interfaces

-Prototyping flexibility 


\subsection{IEEE 1451}

> IEEE Standard for a Smart Transducer Interface for Sensors and Actuators

- Adoption Advantages

-Increased compatibility

-Reduced design effort

-Reduced effort for installation, update, replacement or movement

> Considered but not yet adopted by the avionics domain 


\subsection{IEEE 1451}

To/From user

Network

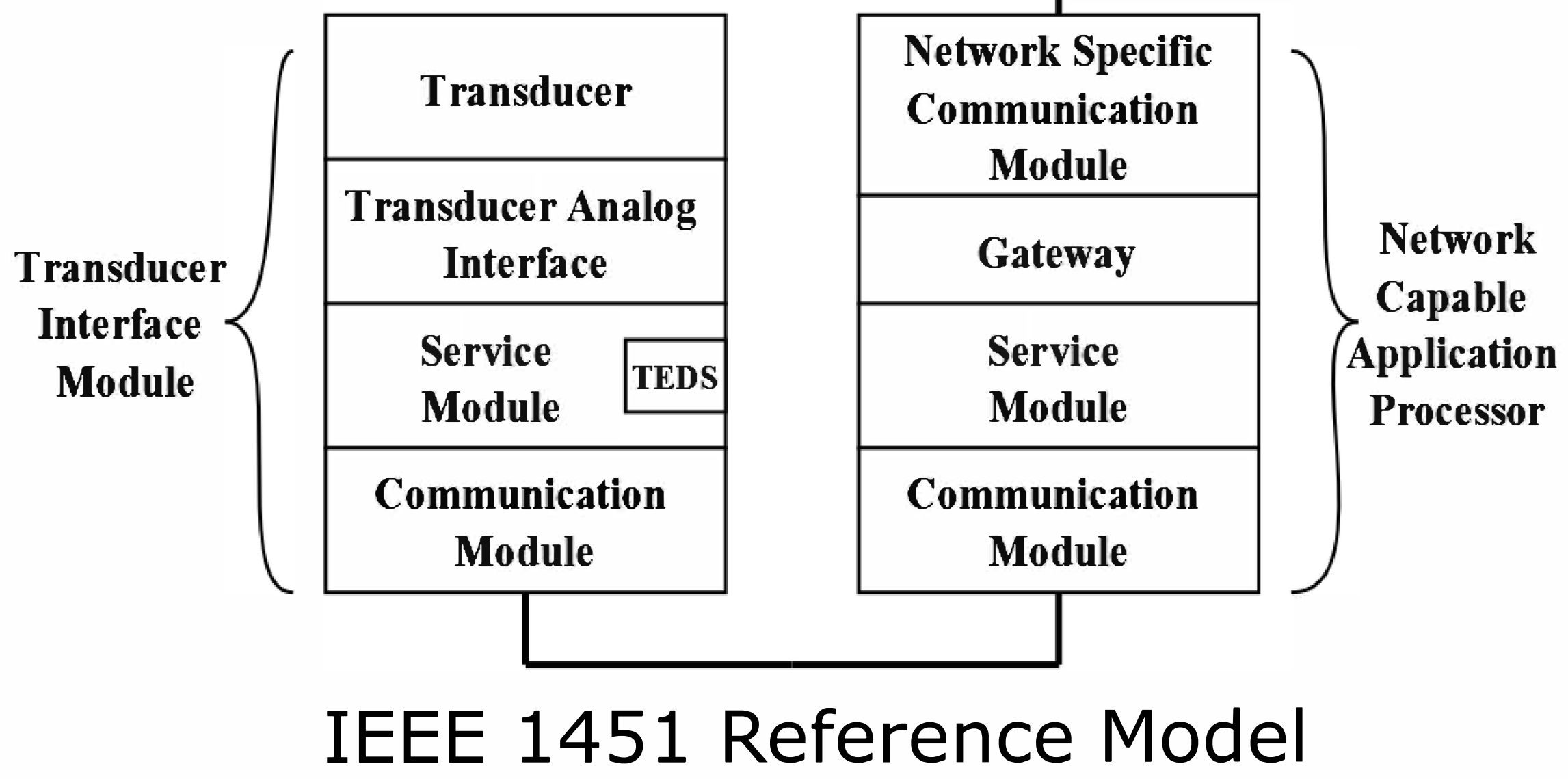




\subsection{Network Architecture}

> Improvements over the basic IEEE 1451

- Improved reliability

- Adjustable by the number of NCAP and Busses

- Improved performances

- Improved resources utilization

- Completely generic for any class of application

-Reconfigurable

Generic Architecture To/From user

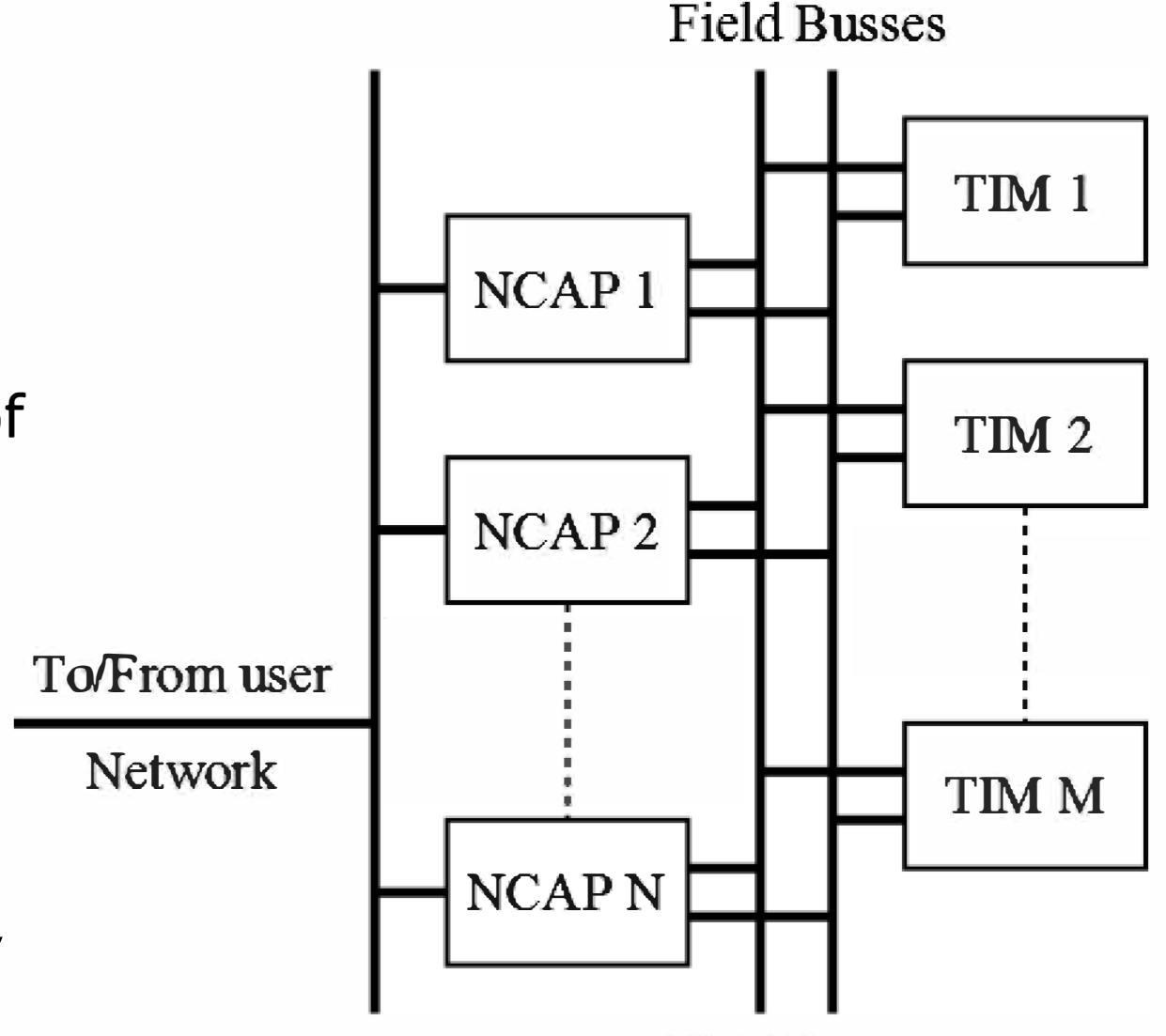

Field Busses 


\subsection{Global approach}

1 Main objective

- Proposition of a systematic approach to validate new technological choices and their integration under important constraints

1 Particular consideration for a compatibility with any certification process such as DO-254/DO-178

- Compatible with current an future design

- Supports new verification constructs

- Tests should be easy to create, maintain and alter

Requirements

Definition

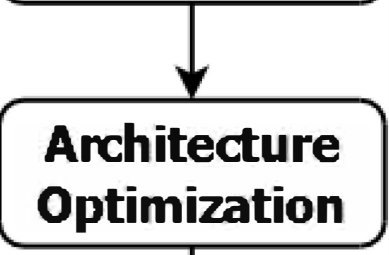

Hardware

Implementation

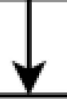

Test Cases

Generation

Prototype Validation 


\subsection{Global approach}

> Requirements definition

- Modeled on traditional avionics requirements

- Architecture optimization

- Generation of a configuration matching the specified requirements

\begin{tabular}{|c|c|}
\hline Requirement & Constraint \\
\hline Failure Rate & $<10 \mathrm{e}^{-\mathbf{6}}$ \\
\hline Load & $<50 \%$ \\
\hline Determinism & Fully Deterministic \\
\hline Frame's Latency & $<2 \mathrm{~ms}$ \\
\hline Bandwidth & $1 \mathrm{Mbit} / \mathrm{s}$ \\
\hline
\end{tabular}

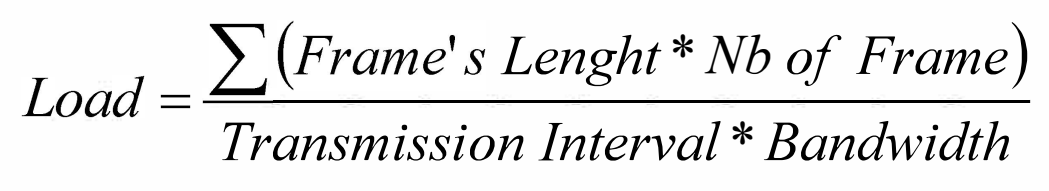

Typical ARINC 825 requirement 


\subsection{Global approach}

) Hardware Implementation

- Connectivity: COTS sensor, transducer emulator, commercial software and PC platform

> Test Cases Generation

- Validation of custom fault management mechanisms

- Supports specific purpose such as any certification process, maintenance or integration of new components

- Motivation behind a custom latency measurement system

- Customized tools best suited for global approach

- Provides a better visualization at the system level 


\section{Outline}

1. Introduction

2. Network architecture

3. Global approach

4. Implementation details

5. Prototype validation

6. Conclusion 


\subsection{Implementation}

- Architecture optimization

- Configuration for the connection of 4 sensors to the main network for a critical system

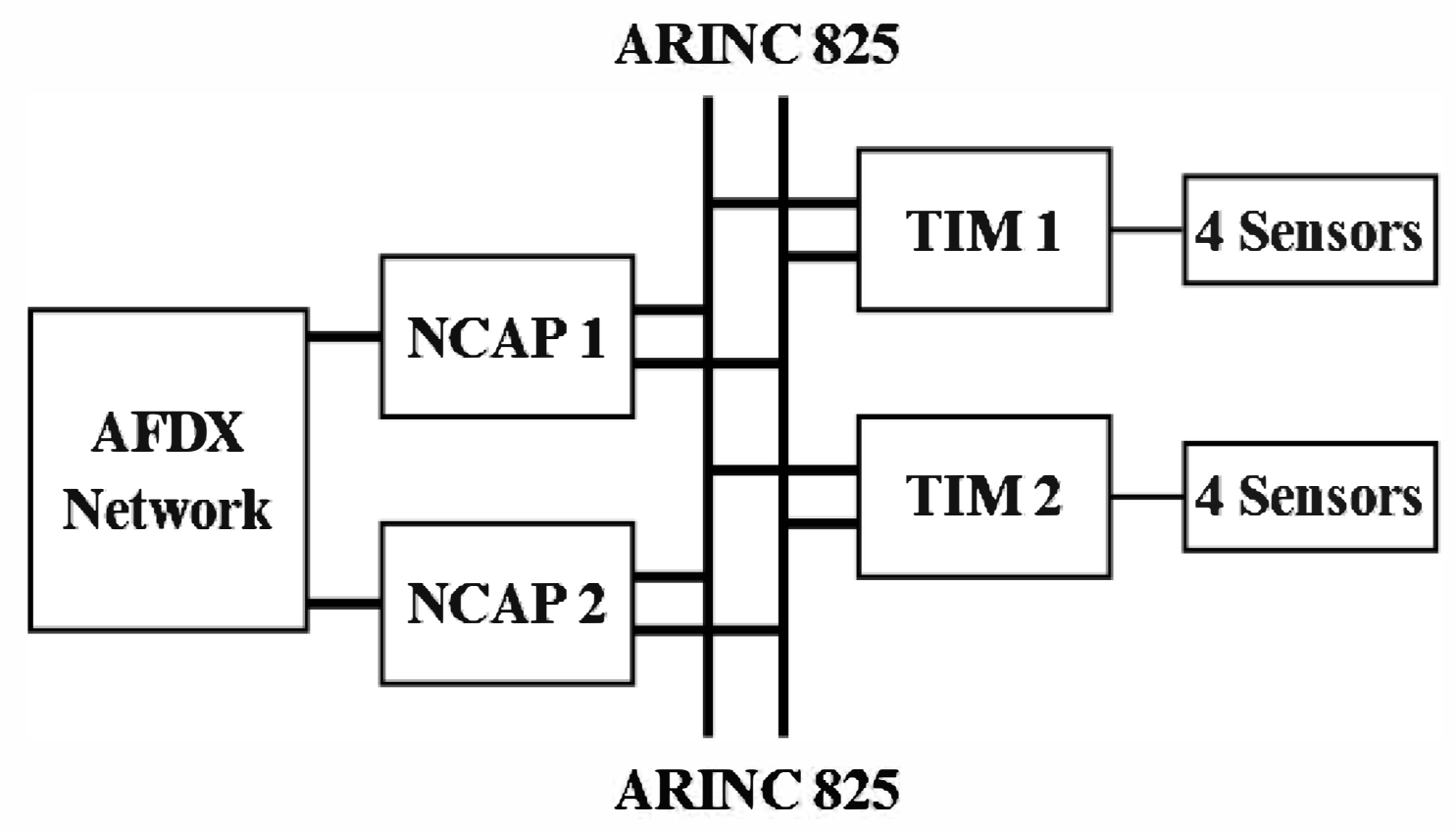

Network architecture 


\subsection{Implementation}

> Prototype platform

-2 SP605 Xilinx FPGA Boards

-2 ISM Networking Boards

> Implementation of selected protocols

-Field bus: ARINC825

- Sensor Interface: I2C

\begin{tabular}{|c|c|c|c|c|}
\hline Module & \multicolumn{2}{|c|}{ LUT } & \multicolumn{2}{c|}{ Registers } \\
\hline $\begin{array}{c}\text { Single } \\
\text { ARINC 825 } \\
\text { Controller }\end{array}$ & 856 & $2 \%$ & 1101 & $4 \%$ \\
\hline $\begin{array}{c}\text { Dual } \\
\text { ARINC 825 } \\
\text { Controller }\end{array}$ & 1831 & $3 \%$ & 2126 & $8 \%$ \\
\hline $\begin{array}{c}\text { TIM's Service } \\
\text { Module }\end{array}$ & 5975 & $11 \%$ & 11198 & $41 \%$ \\
\hline $\begin{array}{c}\text { NCAP's Service } \\
\text { Module }\end{array}$ & 1112 & $2 \%$ & 850 & $3 \%$ \\
\hline TIM & 7806 & $14 \%$ & 13324 & $49 \%$ \\
\hline NCAP & 2943 & $5 \%$ & 2976 & $11 \%$ \\
\hline Total Architecture & 21498 & $10 \%$ & 24522 & $11 \%$ \\
\hline
\end{tabular}

Architecture's complexity 


\subsection{Implementation}

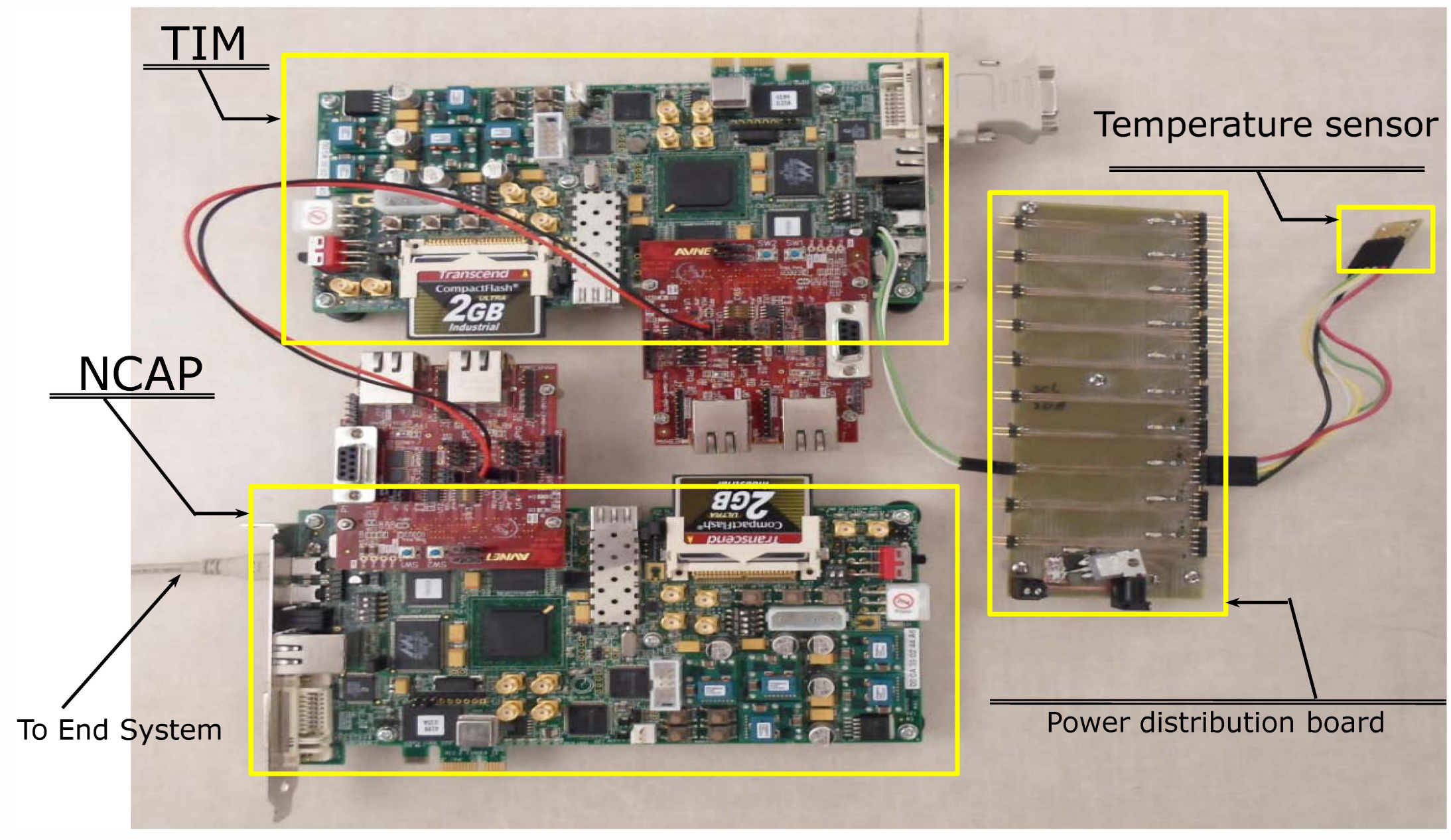




\subsection{Implementation}

1 The VHDL custom implementation allowed the inclusion of novel fault management schemes

> Redundancy management is based on the error containment system

-Bus is shut off upon the degradation on bus on either transmission or reception

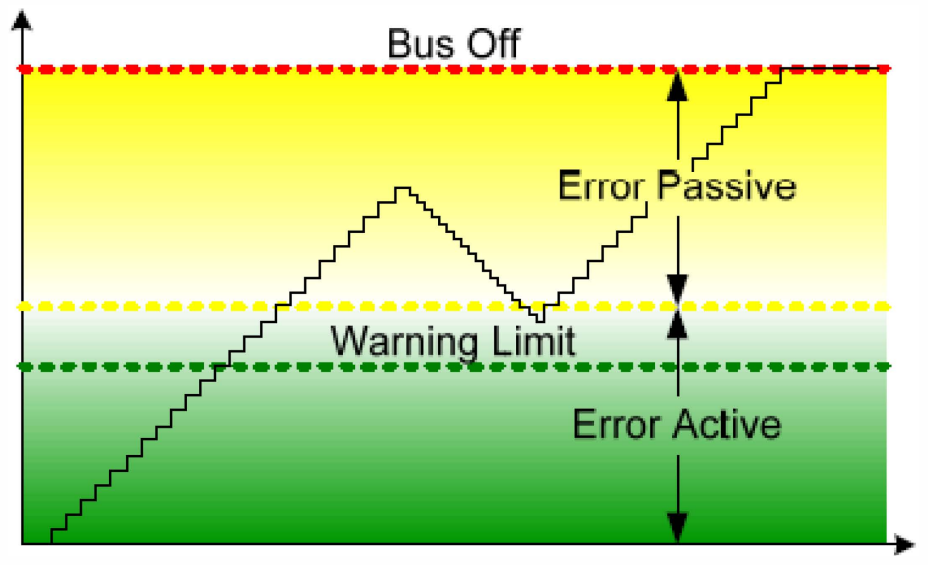

Transmitter Error Count Vs Mode of error

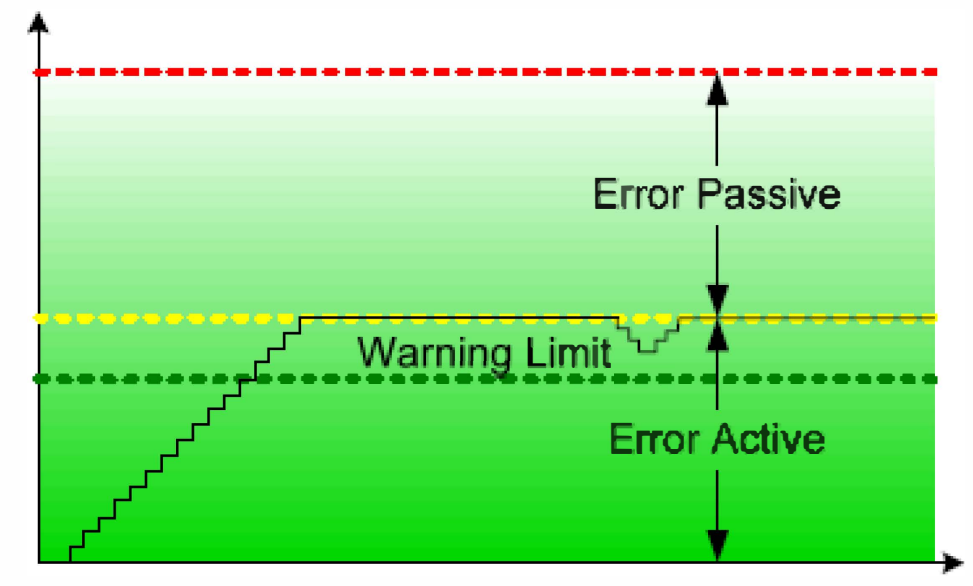

Receiver Error Count Vs Mode of error 


\subsection{Prototype Validation}

1 Test Cases Generation

- Specific test designed for selected requirements at the required level

- Connection with commercial software ADS2

- Bandwidth validation of ARINC 825

- Tests generation at higher levels of abstraction

> Custom latency measurement

-Integrated to the time synchronization mechanism of ARINC 825

- Compensation for extra transmission time due to bit stuffing 


\subsection{Prototype validation}

Validation of the requirements

- The maximum latency is inferior to $2 \mathrm{~ms}$

- The maximum load in normal mode is inferior to $50 \%$

- The slight variation of latency for each frame during each transmission cycle indicates a deterministic traffic

\begin{tabular}{|c|c|c|}
\hline Field Bus Condition & Max Latency (us) & Load \\
\hline Normal & 0.65 & $33 \%$ \\
\hline 1 bus off & 1.05 & $53 \%$ \\
\hline
\end{tabular}

Network Load

\begin{tabular}{|c|c|c|}
\hline Sensor & Latency (Cycle) & Latency (us) \\
\hline 1A & 2698 & 337 \\
\hline 2A & 3342 & 417 \\
\hline 3A & 4421 & 552 \\
\hline 4A & 5090 & 636 \\
\hline 1B & 2699 & 337 \\
\hline 2B & 3594 & 449 \\
\hline 3B & 4432 & 554 \\
\hline 4B & 5104 & 638 \\
\hline
\end{tabular}

Average Frame's Latency 


\subsection{Prototype validation}

- Identification of an unforeseen problem in our custom fault management mechanism

- Upon degradation of a bus, retransmissions caused frames to miss their deadline

- None of our models predicted this worst case situation occurring only during reconfiguration

> Final improvement

- Modification over the original scheme to correct the problem and respect the requirements

- A better knowledge of the standard is helpful in the identification and correction of this problem 


\subsection{Conclusion}

> New approach for design and validation of an avionic network

- The prototyping platform grants an increase connectivity and flexibility

- The proposed approach is compatible with any certification process

> Future work

- Automatic optimization of the architecture under a specific set of constraints

-Validation of new algorithms and novel sensor designs 
6.2 Questions

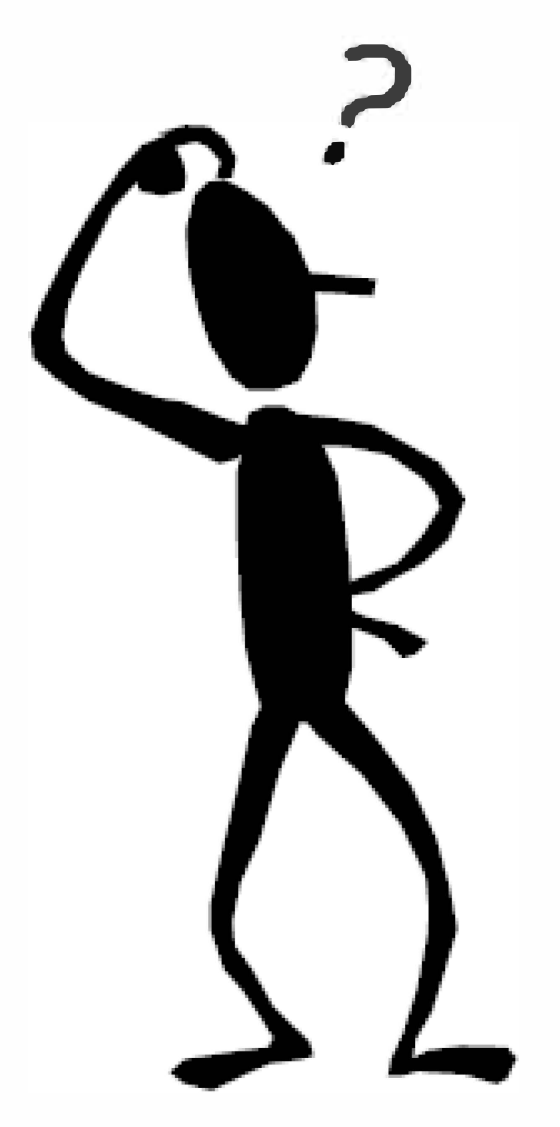

